\title{
LORÍSTICA EM FITOFISIONOMIAS DE RESTINGA NA BAHIA, NORDESTE DO BRASIL
}

\author{
Grênivel Mota da Costa
}

Universidade Federal do Recôncavo da Bahia, Centro de Ciências Agrárias, Ambientais e Biológicas, Cruz das Almas, Bahia, Brasil. E-mail: grenivel@gmail.com

\section{Juliana de Souza Pereira}

Universidade Federal do Recôncavo da Bahia, Centro de Ciências Agrárias, Ambientais e Biológicas, Cruz das Almas, Bahia, Brasil. E-mail: julianasouzaa10@hotmail.com

\section{MÁRCIO LACERda Lopes MARTINS}

Universidade Federal do Recôncavo da Bahia, Centro de Ciências Agrárias, Ambientais e Biológicas, Cruz das Almas, Bahia, Brasil. E-mail: marciollm@ufrb.edu.br

\section{Lidyanne Yuriko Saleme Aona}

Universidade Federal do Recôncavo da Bahia, Centro de Ciências Agrárias, Ambientais e Biológicas, Cruz das Almas, Bahia, Brasil. E-mail: lidyanne.aona@gmail.com

78 Resumo: Teve-se como objetivo no presente estudo teve como objetivo listar as espécies ocorrentes em uma área de restinga em Jaguaripe, Bahia, caracterizando as espécies quanto ao endemismo e distribuição, além de relacioná-las com as distintas fitofisionomias encontradas. As coletas ocorreram periodicamente entre junho de 2014 e agosto de 2017. Na área de estudo, foram coletadas 195 espécies, distribuídas em 150 gêneros e 65 famílias. As famílias mais diversas foram Fabaceae e Rubiaceae, enquanto Myrcia DC. foi o gênero mais representativo. Entre os hábitos encontrados, as herbáceas foram os que apresentaram maior número de espécies (90), seguido por arbustos (61), trepadeiras (24) e árvores com 20 espécies. Estudos florísticos em outras restingas da Bahia têm apontado a ocorrência das mesmas famílias como as de maior riqueza em espécies, entretanto, o número de espécies do presente estudo enquadraram-se entre os mais baixos do estado. Diversas espécies são endêmicas do domínio fitogeográfico da Floresta Atlântica, da restinga ou do estado da Bahia. Cinco espécies são invasoras ou naturalizadas. Os dados obtidos no presente estudo podem subsidiar ações de conservação das restingas da costa do Brasil.

Palavras-chave: Baixo Sul da Bahia, herbário, Jaguaripe, levantamento florístico, Myrcia.

\section{FLORISTICS IN RESTINGA PHYTOPHYSIOGNOMIES IN BAHIA,NORTHEASTERN BRAZIL}

\begin{abstract}
The aim in the present study was to make a list of species occurring in a restinga area in Jaguaripe, Bahia, with data on endemism and distribution, as well as relating them to the different phytophysiognomies found. The collections occurred periodically between June 2014 and August 2017. In the study area, 195 species were collected, distributed in 150 genera and 65 families. The most diverse families were Fabaceae and Rubiaceae, while Myrcia DC. was the most representative genera. Among the habitats found, the herbaceous species had the highest number of species, totaling 90 species, followed by shrubs (61), vines (24) and trees with 20 species. Floristic studies in other restingas of Bahia have indicated the occurrence of the same families as the ones of greater wealth in species, however, the number of species of the present study was among the lowest in the state. Several species are endemic to the phytogeographical domain of the Atlantic Forest, the restinga or the state of Bahia. Five species are invasive or naturalized. The data obtained in the present study can support conservation
\end{abstract}


actions for the restingas.

Keywords: South Bahia, floristic survey, herbarium, Jaguaripe, Myrcia.

\section{INTRODUÇÃo}

A diversidade vegetal brasileira ainda requer esforços de inventários (Martinelli, 2010). Uma forma de incrementar tais dados é através de estudos florísticos, fundamentais para registrar quais espécies de plantas ocorrem em uma determinada localidade, crescentando dados sobre a distribuição de espécies e seus padrões de ocorrência e subsidiando, dessa forma, a compreensão biogeográfica de um bioma (Kendal \& Snelson, 2009; Moro \& Martins, 2011). Tais estudos, ainda permitem inúmeras análises botânicas e ecológicas, ferramentas para caracterizar a vegetação, o que pode ser útil na delimitação e conservação de comunidades vegetais com distintas fitofisionomias ou habitats (Kendal \& Snelson, 2009; Moro \& Martins, 2011), como por exemplo, a restinga.

As restingas são tipos vegetacionais associados aos depósitos arenosos costeiros com influência flúvio-marinha (Meira-Neto et al., 2005; Magnago et al., 2010), geralmente relacionados ao Domínio Fitogeográfico da Mata Atlântica, que por sua vez é considerada um hotspot de biodiversidade (Myers et al., 2000). Variações nas condições do solo, da geomorfologia, da topografia, do nível do lençol freático e do gradiente de vegetação mar-interior possibilitam a existência de comunidades de restinga que são fisionômica e floristicamente distintas (Araújo, 2000; Magnago et al., 2010; Martins, 2012; Saporetti-Junior et al., 2012). A forte influência da proximidade com florestas pluviais é evidenciada nesse tipo de vegetação (Fernandes \& Queiroz, 2015), além da disjunção florística do componente herbáceo com os campos rupestres (Harley, 1995).

Estudos florísticos em restingas têm apontado aproximadamente 370 espécies endêmicas (BFG, 2015), dentre elas algumas ameaçadas de extinção ou invasoras, dados estes relevantes que podem ser relacionados diretamente com ações de conservação, visto a forte pressão antrópica sobre as restingas através da alta especulação imobiliária e turística, remoção de areia e abertura de estradas (Fanzeres, 2003; Rocha et al., 2007).

A composição florística das restingas é melhor conhecida nos estados do Espírito Santo e Rio de Janeiro (Pereira et al., 1992; Pereira \& Araújo, 1995; Fabris \& Pereira, 1998; Araujo et al., 2004; Rocha et al., 2007; Colodete \& Pereira, 2007; Magnago et al., 2011a). Para a Bahia, o conhecimento desta flora tem se intensificado nos últimos anos, evidenciando sua composição florística (Meira-Neto et al., 2005; Queiroz et al., 2012; Fernandes \& Queiroz, 2015) ou puramente aspectos fisionômicos (Meira-Neto et al. 2005, Martins 2012). Entretanto, para uma melhor resolução e possibilidade de análises mais refinadas, novos estudos sobre composição florística desse tipo de vegetação são necessários. Dessa forma, no presente estudo teve-se como objetivo listar as espécies ocorrentes numa área de restinga em Jaguaripe, Bahia, caracterizando o endemismo e distribuição dessas espécies, relacioná-las com as distintas fitofisionomias, bem como avaliar a similaridade com outras áreas de restinga ao longo do litoral brasileiro.

\section{Material e métodos}

\section{ÁreA DE ESTUdo}

O estudo foi conduzido no município de Jaguaripe, Bahia, localizado na região Baixo-Sul do estado. O Baixo Sul caracteriza-se por apresentar clima tropical com elevadas temperaturas e precipitações, influenciadas pela proximidade do mar. As temperaturas médias anuais variam entre $21^{\circ}$ e $25^{\circ} \mathrm{C}$, a pluviosidade é regular com chuvas abundantes distribuídas durante o ano, com médias anuais superiores a $1.750 \mathrm{~mm}$ (SEI, 2015). Os meses de maior precipitação estão entre março e junho, enquanto os de menor precipitação entre agosto e outubro, a umidade relativa média gira em torno de 80 a $90 \%$, e a velocidade média dos ventos varia entre $1,29 \mathrm{~m} / \mathrm{s}$ a $2,9 \mathrm{~m} / \mathrm{s}$ dependendo dos meses do ano (SEI, 2015). A área não possui uma estação seca definida e o solo apresenta-se como Podzol Hidromórfico e Areias Quartzosas marinhas (SEI, 2015).

A área estudada compreende os dois cordões arenosos mais próximos à praia, 


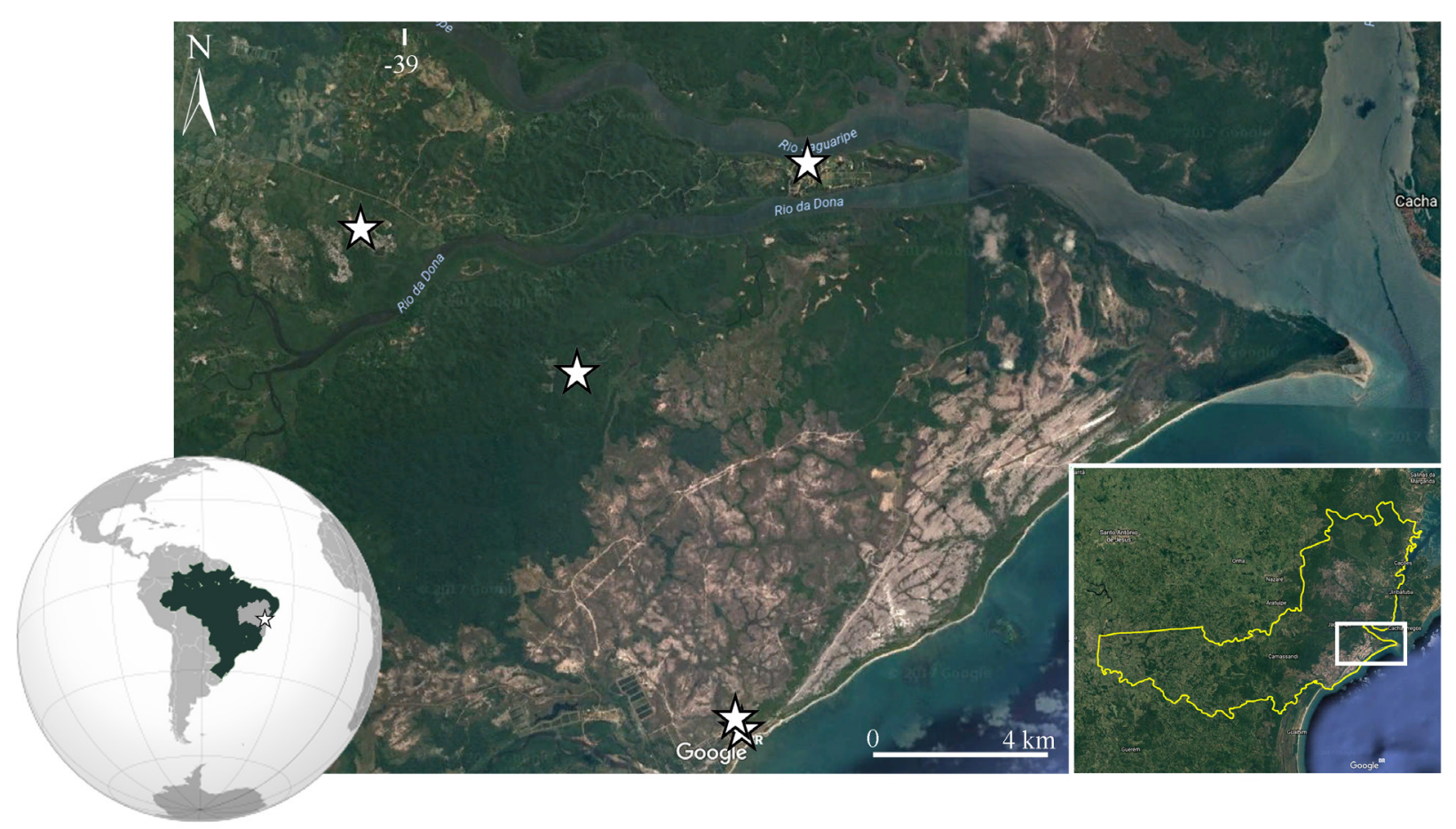

Fig. 1. Restinga estudada, com seus respectivos pontos de coleta (estrela), em Jaguaripe, Bahia, Brasil. (Imagem obtida através do GoogleEarth.)

com extensão de $4 \mathrm{~km}$, limitados pelo Rio da Dona (Fig. 1). O primeiro cordão apresenta largura aproximada de $100 \mathrm{~m}$ e está coberto de vegetação herbácea e arbustiva nãoinundável (VNI), moderadamente impactada por empreendimentos imobiliários. A depressão que separa os dois cordões é ocupada por vegetação herbácea e arbustiva inundável (VI), margeada por estradas de acesso às áreas residenciais. O segundo cordão estende-se por cerca de $200 m$ de largura com vegetação ainda preservada. Após este trecho, a vegetação foi substituída por loteamentos imobiliários. Essa área está coberta por vegetação que aumenta de porte a medida que o terreno se eleva, partindo da fisionomia herbácea inundável, com ilhas de vegetação arbustiva inundável e não inundável (IV) com espécies de até $3 \mathrm{~m}$ de altura e chegando à floresta inundável (FI) com espécies que alcançam cerca de $6 \mathrm{~m}$ de altura (Fig. 2). A caracterização das fisionomias foi adaptada de Martins (2012) e Pereira (2003).

\section{LEVANTAMENTO FLORÍSTICO}

As coletas ocorreram trimestralmente entre junho de 2014 e agosto de 2017. Foram coletados e herborizados materiais férteis, com flores e/ou frutos segundo Mori et al. (1989). A identificação do material foi feita com base em bibliografia especializada, comparação em herbário e consulta direta aos especialistas. O sistema de classificação adotado foi o APG IV (2016) e os nomes das espécies são apresentados conforme a Lista de Espécies da Flora do Brasil (BFG, 2015; Flora do Brasil 2020 em construção), onde também foram retiradas informações acerca da distribuição, status de conservação e endemismo das espécies. Quando estas informações mostravam-se dúbias ou insuficientes, buscaram-se informações em bases de dados de herbários disponíveis no Splink (http://www.splink.org.br) e no Reflora (Flora do Brasil 2020 em construção). Todo o material foi depositado no Herbário do Recôncavo da Bahia (HURB).

\section{ANÁLISE DE SIMILARIDADE}

A partir de 15 levantamentos florísticos em áreas de restinga foi elaborada uma matriz de presença e ausência de espécies para avaliar similaridade (Tab. 1). Na matriz foram incluídos apenas táxons identificados até o nível de espécie, excluindo-se aquelas com identificação imprecisa (assinaladas com 'cf.' ou 'aff.'). Foi utilizada a análise de Cluster utilizando o método de WPGMA utilizando o coeficiente de Soresen (Magurran, 2003) como medida de similaridade, as espécies que ocorriam em apenas uma localidade foram excluídas da análise. A análise foi processada com uso do programa PAST (Hammer et al., 2001). 

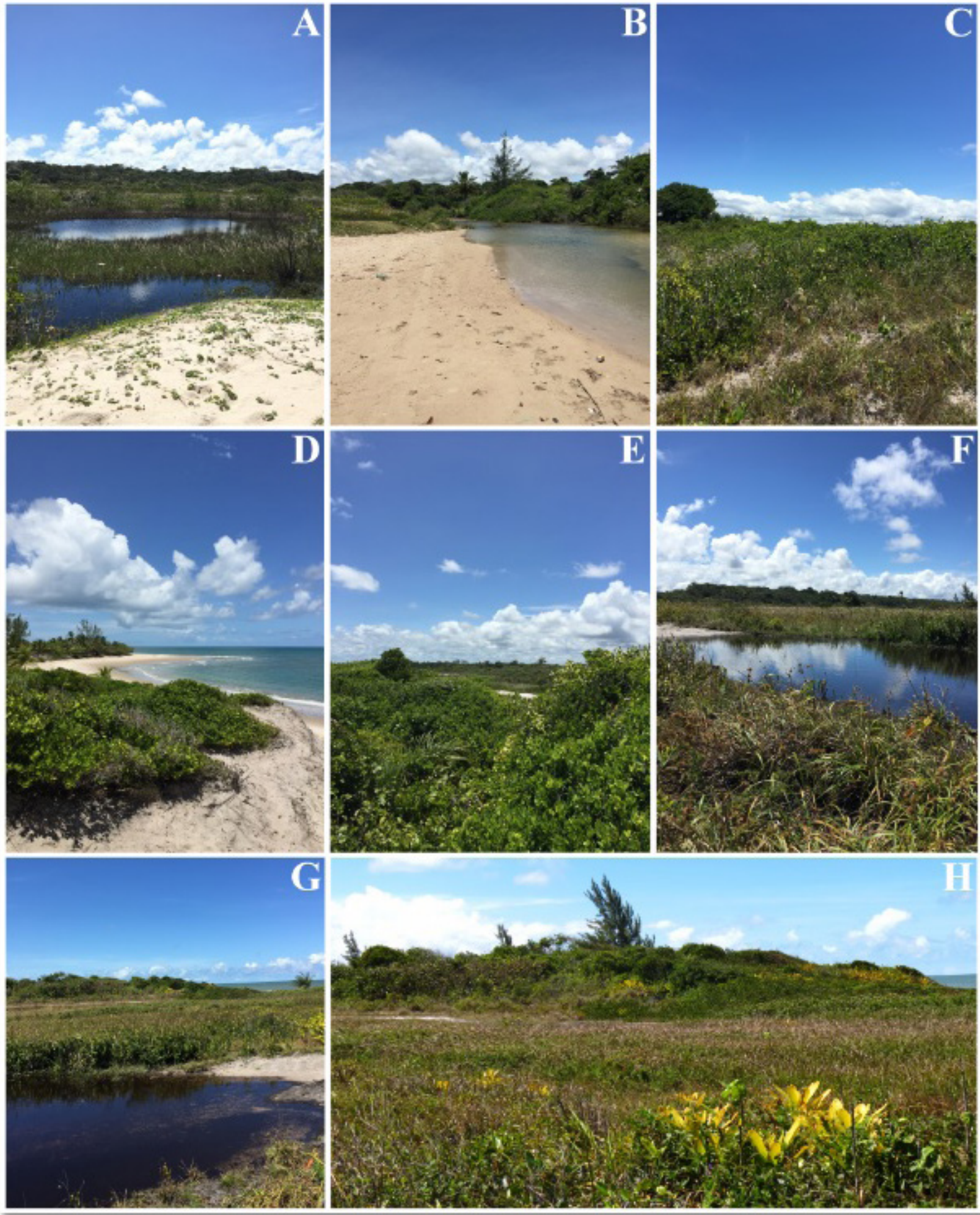

Fig. 2. Fisionomias amostradas na restinga de Jaguaripe, Bahia, Brasil. A - vegetação herbácea e arbustiva inundável (VI); B - rio da Dona que corta a restinga na com floresta inundável (FI) ao fundo; C-D - vegetação herbácea e arbustiva não-inundável (VNI); E - ilhas de vegetação arbustiva inundável e não inundável (IV); F - vegetação herbácea e arbustiva não-inundável (VNI); G - vegetação herbácea e arbustiva inundável (VI) com ilhas de vegetação arbustiva inundável e não inundável (IV) ao fundo; $\mathrm{H}$ - aspecto geral da restinga. 
Tab. 1. Áreas de restinga usadas na análise Cluster, com sigla, tipos de vegetação, localização e autoria.

\begin{tabular}{|c|c|c|c|}
\hline Sigla & Tipo de vegetação & Município/Estado & Autores \\
\hline ResManRJ & Restinga arbustiva & $\begin{array}{c}\text { Arraial do Cabo, Rio de } \\
\text { Janeiro }\end{array}$ & Carvalho \& Sá 2011 \\
\hline ResBarRJ & Restinga arbustiva & $\begin{array}{c}\text { Barra do Maricá, Rio de } \\
\text { Janeiro }\end{array}$ & Pereira et al. 2001 \\
\hline ResGuaPE & $\begin{array}{l}\text { Distintas fisionomias de } \\
\text { restinga }\end{array}$ & Sirinhaém, Pernambuco & Cantarelli et al. 2012 \\
\hline ResSanSE & Não especificado & $\begin{array}{c}\text { Pirambu e Pacatuba, } \\
\text { Sergipe }\end{array}$ & Oliveira et al. 2015 \\
\hline ResItaES & Restinga Arbustiva & $\begin{array}{l}\text { Conceição da Barra, } \\
\text { Espírito Santo }\end{array}$ & Monteiro et al. 2014 \\
\hline ResMaBA & Restinga Arbórea & Maraú, Bahia & Fernandes \& Queiroz 2015 \\
\hline ResIlhPI & $\begin{array}{l}\text { Distintas fisionomias de } \\
\text { restinga }\end{array}$ & Ilha Grande, Piauí & Santos-Filho 2009 \\
\hline ResParPI & Restinga herbácea & Parnaíba, Piauí & Santos-Filho 2009 \\
\hline ResLuiPI & Restinga Arbustiva & Luiz Correia, Piauí & Santos-Filho 2009 \\
\hline ResCamBA & Diversas fisionomias & Camaçari, Bahia & Queiroz et al. 2012 \\
\hline ResMatBA & $\begin{array}{l}\text { Floresta de Restinga e } \\
\text { Ilhas de vegetação }\end{array}$ & Mata de São João, Bahia & Menezes et al. 2012 \\
\hline ResGuaES & Restinga Arbórea & Guarapari, Espírito Santo & Assis et al. 2004 \\
\hline ResCamBA & Diversas fisionomias & Camaçari, Bahia & Queiroz et al 2012 \\
\hline ResMatBA & $\begin{array}{l}\text { Floresta de Restinga e } \\
\text { Ilhas de vegetação }\end{array}$ & Mata de São João, Bahia & Menezes et al 2012 \\
\hline ResGuaES & Restinga Arbórea & Guarapari, Espírito Santo & Assis et al 2004 \\
\hline
\end{tabular}

\section{Resultados}

Levantamento florístico - Na área de estudo, foram coletadas 195 espécies distribuídas em 150 gêneros e 65 famílias. A razão entre o número de espécies e número de gêneros é de 1,3. As famílias mais diversas foram Fabaceae com 20 espécies e Rubiaceae com 19 espécies, seguidas por Melastomataceae (11) e Eriocaulaceae (10) (Tab. 2). O gênero Myrcia DC. (Myrtaceae) foi o mais diverso na restinga de Jaguaripe, com quatro espécies.

Tab. 2. Lista de plantas em fisionomias de restinga em Jaguaripe, Bahia. FC - Forma de crescimento; End. Endemismo; DFB - Domínio Fitogeográfico Brasileiro; ER - Erva; AB - Arbusto; AV- Árvore; LI - Liana; VNI - vegetação herbácea e arbustiva não-inundável; VI - vegetação herbácea e arbustiva inundável; IV - ilhas de vegetação arbustiva inundável e não inundável; FI - floresta inundável; NE - Espécie não endêmica do Brasil; EE - Espécie endêmica do Brasil; DE - Desconhecido; AM - Domínio Fitogeográfico Amazônico; CA Domínio Fitogeográfico da Caatinga; CE - Domínio Fitogeográfico do Cerrado; PT - Domínio Fitogeográfico do Pantanal; MA - Domínio Fitogeográfico da Floresta Atlântico; PM - Domínio Fitogeográfico do Pampa.

* - espécie ameaçada de extinção; ** - espécie invasora; *** - espécie naturalizada; espécies endêmicas da restinga estão marcadas em negrito.

\begin{tabular}{ccccccc}
\hline Família & Espécie & Tombo & FV & $\begin{array}{c}\text { Fisiono- } \\
\text { mia }\end{array}$ & End. & DFB \\
\hline & & & & & & \\
\hline Acanthaceae & Ruellia geminiflora Kunth. & 15423 & ER & VI & NE & AM/CA/CE/PT \\
\hline
\end{tabular}


TAB. 2 - CONTINUAÇÃO

\begin{tabular}{|c|c|c|c|c|c|c|}
\hline Anacardiaceae & Schinus terebinthifolius Raddi & 8567 & $A B$ & IV/VNI & $\mathrm{NE}$ & CE/MA/PM \\
\hline Anacardiaceae & Tapirira guianensis Aubl. & 9339 & AV & FI/IV/VNI & $\mathrm{NE}$ & $\begin{array}{l}\text { AM/CA/CE/ } \\
\text { MA/PM/PT }\end{array}$ \\
\hline Annonaceae & Annona glabra L. & 15310 & AV & VNI & NE & AM/MA \\
\hline Annonaceae & Guatteria ferruginea A.St.-Hil. & 7886 & $A V$ & FI & $\mathrm{EE}$ & MA \\
\hline Apocynaceae & Blepharodon ampliflorum E.Fourn & 7854 & LI & VNI & $\mathrm{EE}$ & $\mathrm{CA} / \mathrm{CE} / \mathrm{MA}$ \\
\hline Apocynaceae & $\begin{array}{c}\text { Blepharodon pictum (Vahl) } \\
\text { W.D.Stevens }\end{array}$ & 9298 & LI & VNI & NE & AM/CA/CE/MA \\
\hline Apocynaceae & $\begin{array}{l}\text { Mandevilla scabra (Hoffmanns. } \\
\text { ex Roem. \& Schult.) K.Schum. }\end{array}$ & 9333 & LI & IV/VNI & $\mathrm{NE}$ & $\mathrm{AM} / \mathrm{CA} / \mathrm{CE} / \mathrm{MA}$ \\
\hline Aquifoliaceae & Ilex psammophila Mart. ex Reissek & 15082 & $A B$ & IV & $\mathrm{EE}$ & MA \\
\hline Araceae & Philodendron acutatum Schott & 9313 & LI & VI & $\mathrm{NE}$ & $\mathrm{AM} / \mathrm{CA} / \mathrm{CE} / \mathrm{MA}$ \\
\hline Araceae & $\begin{array}{c}\text { Dracontioides desciscens (Schott) } \\
\text { Engl. }\end{array}$ & 15402 & $\mathrm{ER}$ & VI & $\mathrm{EE}$ & MA \\
\hline Araliaceae & $\begin{array}{l}\text { Hydrocotyle leucocephala Cham. \& } \\
\text { Schldl. }\end{array}$ & 15728 & ER & VNI & $\mathrm{NE}$ & $\mathrm{CE} / \mathrm{MA}$ \\
\hline Asteraceae & $\begin{array}{l}\text { Bahianthus viscosus (Spreng.) } \\
\text { R.M. King \& H. Rob. }\end{array}$ & 11304 & $A B$ & IV/VI & $\mathrm{EE}$ & $\mathrm{CA} / \mathrm{CE} / \mathrm{MA}$ \\
\hline Asteraceae & Calea angusta S.F. Blake & 9349 & $A B$ & VI & $\mathrm{DE}$ & $\mathrm{CE} / \mathrm{CA}$ \\
\hline Asteraceae & $\begin{array}{c}\text { Cyrtocymura scorpioides (Lam.) } \\
\text { H.Rob. }\end{array}$ & 15047 & $A B$ & VI & $\mathrm{EE}$ & AM/CE \\
\hline Asteraceae & Elephantopus hirtiflorus DC. & 11021 & $\mathrm{ER}$ & VI & $\mathrm{EE}$ & $\mathrm{CA} / \mathrm{CE} / \mathrm{MA}$ \\
\hline Asteraceae & $\begin{array}{c}\text { Eremanthus capitatus (Spreng.) } \\
\text { MacLeish }\end{array}$ & 7871 & AV & IV/VNI & $\mathrm{EE}$ & $\mathrm{CA} / \mathrm{CE} / \mathrm{MA}$ \\
\hline Asteraceae & $\begin{array}{c}\text { Lepidaploa edmundoi (G.M.Barroso) } \\
\text { H.Rob. }\end{array}$ & 15073 & $A B$ & IV/VI & EE & $\mathrm{CA} / \mathrm{CE}$ \\
\hline Asteraceae & $\begin{array}{l}\text { Prolobus nitidulus (Baker) } \\
\text { R.M.King \& H.Rob. }\end{array}$ & 15035 & $A B$ & VNI & $\mathrm{EE}$ & MA \\
\hline Bonnetiaceae & Bonnetia stricta (Nees) Nees \& Mart. & 7911 & $A B$ & IV/VI & $\mathrm{EE}$ & $\mathrm{CA} / \mathrm{MA}$ \\
\hline Boraginaceae & Varronia curassavica Jacq. & 9310 & $A B$ & VNI & $\mathrm{NE}$ & $\mathrm{AM} / \mathrm{CA} / \mathrm{CE} / \mathrm{MA}$ \\
\hline Bromeliaceae & $\begin{array}{c}\text { Aechmea blanchetiana (Baker) } \\
\text { L.B.Sm.* }\end{array}$ & 9325 & $\mathrm{ER}$ & VI & $\mathrm{EE}$ & MA \\
\hline Bromeliaceae & $\begin{array}{l}\text { Aechmea mertensii (G. Mey.) } \\
\text { Schult. \& Schult.f. }\end{array}$ & 11000 & ER & VNI & $\mathrm{NE}$ & AM/MA \\
\hline Bromeliaceae & Vriesea neoglutinosa Mez & 7912 & ER & IV & $\mathrm{EE}$ & MA \\
\hline Burmanniaceae & $\begin{array}{l}\text { Burmannia capitata (Walter ex J.F. } \\
\text { Gmel.) Mart. }\end{array}$ & 11012 & ER & VI & $\mathrm{NE}$ & AM/CE/MA \\
\hline Calophyllaceae & Calophyllum brasiliense Cambess. & 9323 & AV & VNI & $\mathrm{NE}$ & $\mathrm{AM} / \mathrm{CA} / \mathrm{CE} / \mathrm{MA}$ \\
\hline Calophyllaceae & Kielmeyera neglecta Saddi & 7861 & AV & VNI & EE & MA \\
\hline Casuarinaceae & Casuarina equisetifolia L. & 15036 & AV & VNI & $\mathrm{NE}$ & - \\
\hline
\end{tabular}




\begin{tabular}{|c|c|c|c|c|c|c|}
\hline Celastraceae & $\begin{array}{c}\text { Maytenus distichophylla Mart. ex } \\
\text { Reissek }\end{array}$ & 15038 & $A B$ & IV & EE & MA \\
\hline $\begin{array}{l}\text { Chrysobalana- } \\
\text { ceae }\end{array}$ & Chrysobalanus icaco L. & 9301 & $A B$ & VNI & NE & AM/MA \\
\hline Clusiaceae & Clusia sellowiana Schltdl. & 7857 & $A B$ & FI/IV & EE & MA \\
\hline Clusiaceae & Symphonia globurifera L.f. & 9347 & AV & FI & NE & $\mathrm{AM} / \mathrm{CE} / \mathrm{MA}$ \\
\hline Combretaceae & Conocarpus erectus L. & 15305 & $A B$ & VI & NE & AM/MA \\
\hline Combretaceae & $\begin{array}{c}\text { Laguncularia racemosa (L.) } \\
\text { C.F.Gaertn. }\end{array}$ & 15303 & AV & VI & NE & AM/MA \\
\hline Convolvulaceae & $\begin{array}{c}\text { Daustinia montana (Moric.) Buril \& A. } \\
\text { R. Simões }\end{array}$ & 9312 & LI & IV/VNI & EE & $\mathrm{CA} / \mathrm{MA}$ \\
\hline Convolvulaceae & $\begin{array}{c}\text { Ipomoea bahiensis Willd. ex Roem. } \\
\text { \& Schult. }\end{array}$ & 15421 & LI & VNI & $\mathrm{EE}$ & $\mathrm{AM} / \mathrm{CA} / \mathrm{CE} / \mathrm{MA}$ \\
\hline Convolvulaceae & Ipomoea imperati (Vahl.) Griseb. & 15311 & LI & VNI & NE & AM/MA \\
\hline Convolvulaceae & Ipomoea pes-caprae (L.) R.Br. & 15422 & LI & VNI & NE & AM/MA \\
\hline Cyperaceae & Becquerelia cymosa Brongn. & 15419 & ER & FI & NE & AM/CE/MA \\
\hline Cyperaceae & Eleocharis minima Kunth & 11520 & ER & VI & NE & $\begin{array}{l}\text { AM/CA/CE/ } \\
\text { MA/PM/ }\end{array}$ \\
\hline Cyperaceae & $\begin{array}{c}\text { Eleocharis atropurpurea (Retz.) } \\
\text { J.Presl \& C.Presl }\end{array}$ & 15572 & ER & VI & EE & $\mathrm{AM} / \mathrm{CA}$ \\
\hline Cyperaceae & Fimbristylis cymosa $\mathrm{R} . \mathrm{Br}$ & 7909 & ER & VNI & $\mathrm{NE}$ & $\mathrm{AM} / \mathrm{CA} / \mathrm{CE} / \mathrm{MA}$ \\
\hline Cyperaceae & Kyllinga brevifolia Rottb. & 15053 & ER & VI & NE & $\begin{array}{l}\text { AM/CA/CE/ } \\
\text { MA/PM/PT }\end{array}$ \\
\hline Cyperaceae & Lagenocarpus rigidus Nees & 9315 & $\mathrm{ER}$ & VI & NE & $\begin{array}{l}\text { AM/CA/CE/ } \\
\text { MA/PT }\end{array}$ \\
\hline Cyperaceae & Scleria latifolia Sw. & 10980 & $\mathrm{ER}$ & VNI & NE & $\begin{array}{l}\mathrm{AM} / \mathrm{CA} / \mathrm{CE} / \\
\mathrm{MA} / \mathrm{PT}\end{array}$ \\
\hline Dilleniaceae & Curatella americana L. & 7860 & $A B$ & VNI & NE & $\mathrm{AM} / \mathrm{CA} / \mathrm{CE} / \mathrm{MA}$ \\
\hline Dilleniaceae & Davilla flexuosa A.St.-Hil. & 9326 & LI & VNI & $\mathrm{EE}$ & MA \\
\hline Eriocaulaceae & $\begin{array}{l}\text { Actinocephalus herzogii var. humilis } \\
\text { (Sano) Sano }\end{array}$ & 8570 & $\mathrm{ER}$ & VI/VNI & $\mathrm{EE}$ & CE \\
\hline Eriocaulaceae & Eriocaulon cf. linearifoliun Körn. & 10989 & $\mathrm{ER}$ & VI & $\mathrm{EE}$ & $\mathrm{CA} / \mathrm{CE}$ \\
\hline Eriocaulaceae & Leiothrix flavescens (Bong) Ruhland & 7865 & ER & VI & NE & $\mathrm{AM} / \mathrm{CA} / \mathrm{CE} / \mathrm{MA}$ \\
\hline Eriocaulaceae & Paepalanthus bifidus (Schrad.) Kunth & 10983 & ER & VI & NE & $\mathrm{AM} / \mathrm{CA} / \mathrm{CE} / \mathrm{MA}$ \\
\hline Eriocaulaceae & $\begin{array}{c}\text { Paepalanthus myocephalus (Mart.) } \\
\text { Körn. }\end{array}$ & 10987 & ER & VI & $\mathrm{EE}$ & $\mathrm{CA} / \mathrm{MA}$ \\
\hline Eriocaulaceae & Paepalanthus sp. & 10991 & ER & VI & 0 & \\
\hline Eriocaulaceae & Paepalanthus sp. & 11025 & ER & VI & 0 & \\
\hline Eriocaulaceae & $\begin{array}{l}\text { Syngonanthus cf. nitens (Bong.) } \\
\text { Ruhland }\end{array}$ & 10998 & $\mathrm{ER}$ & VI & NE & $\mathrm{AM} / \mathrm{CA} / \mathrm{CE} / \mathrm{MA}$ \\
\hline
\end{tabular}




\begin{tabular}{|c|c|c|c|c|c|c|}
\hline Eriocaulaceae & Tonina fluviatilis Aubl. & 7913 & ER & VI & NE & AM/CA/MA \\
\hline Euphorbiaceae & Croton glandulosus L. & 11308 & $A B$ & VNI & NE & $\begin{array}{l}\text { AM/CA/CE/ } \\
\text { MA/PM/PT }\end{array}$ \\
\hline Euphorbiaceae & Croton sellowii Baill. & 7872 & $A B$ & VNI & EE & $\mathrm{CA} / \mathrm{MA}$ \\
\hline Euphorbiaceae & Euphorbia sp. & 11303 & ER & VNI & & \\
\hline Euphorbiaceae & $\begin{array}{l}\text { Microstachys corniculata (Vahl) Gri- } \\
\text { seb. }\end{array}$ & 11305 & ER & VNI & NE & $\mathrm{AM} / \mathrm{CA} / \mathrm{CE} / \mathrm{MA}$ \\
\hline Fabaceae & Abarema filamentosa (Benth.) Pittier & 9307 & AV & IV & EE & MA \\
\hline Fabaceae & $\begin{array}{c}\text { Aeschynomene sensitiva Sw. var. } \\
\text { sensitiva }\end{array}$ & 7856 & $A B$ & VI & NE & AM/CE/MA/PT \\
\hline Fabaceae & $\begin{array}{c}\text { Albizia polycephala (Benth.) Killip ex } \\
\text { Record }\end{array}$ & 7873 & $\mathrm{AV}$ & FI & $\mathrm{EE}$ & $\begin{array}{l}\text { AM/CA/CE/ } \\
\text { MA/PP }\end{array}$ \\
\hline Fabaceae & Centrosema brasilianum (L.) Benth. & 15414 & LI & IV & NE & $\begin{array}{l}\text { AM/CA/CE/ } \\
\text { MA/PP/PT }\end{array}$ \\
\hline Fabaceae & $\begin{array}{l}\text { Chamaecrista desvauxii (Collad.) } \\
\text { Killip }\end{array}$ & 15050 & ER & VNI & NE & $\begin{array}{l}\text { AM/CA/CE/ } \\
\text { MA/PT }\end{array}$ \\
\hline Fabaceae & Chamaecrista flexuosa L. Greene & 15412 & ER & VNI & NE & $\begin{array}{l}\text { AM/CA/CE/ } \\
\text { MA/PP/PT }\end{array}$ \\
\hline Fabaceae & $\begin{array}{c}\text { Chamaecrista ramosa (Vogel) } \\
\text { H.S.Irwin \& Barneby }\end{array}$ & 7866 & ER & VNI & NE & $\begin{array}{l}\text { AM/CA/CE/ } \\
\text { MA/PT }\end{array}$ \\
\hline Fabaceae & Crotalaria retusa L.*** & 7879 & ER & VI & NE & $\begin{array}{l}\text { AM/CA/CE/ } \\
\text { MA/PP }\end{array}$ \\
\hline Fabaceae & $\begin{array}{c}\text { Desmodium adscendens (Sw.) } \\
\text { DC.*** }\end{array}$ & 11009 & ER & VNI & NE & $\begin{array}{l}\text { AM/CA/CE/ } \\
\text { MA/PP }\end{array}$ \\
\hline Fabaceae & Desmodium barbatum (L.) Benth. & 11011 & ER & VNI & NE & $\begin{array}{l}\text { AM/CA/CE/ } \\
\text { MA/PP/PT }\end{array}$ \\
\hline Fabaceae & Desmodium triflorum (L.) DC.*** & 7882 & ER & VNI & NE & $\begin{array}{l}\text { AM/CA/CE/ } \\
\text { MA/PT }\end{array}$ \\
\hline Fabaceae & Dioclea lasiophylla Mart. Ex Benth. & 11290 & $\mathrm{LI}$ & IV & EE & $\mathrm{CA} / \mathrm{MA}$ \\
\hline Fabaceae & Hymenolobium alagoanum Ducke & 11289 & $A B$ & IV & EE & MA \\
\hline Fabaceae & $\begin{array}{c}\text { Mimosa somnians Humb. \& Bonpl. ex } \\
\text { Willd. }\end{array}$ & 15720 & $A B$ & IV & & AM/CA/CE/PT \\
\hline Fabaceae & Periandra mediterranea (Vell.) Taub. & 7877 & $A B$ & IV & NE & $\mathrm{AM} / \mathrm{CA} / \mathrm{CE} / \mathrm{MA}$ \\
\hline Fabaceae & Rhynchosia minima (L.) DC. & 11284 & LI & IV & NE & $\begin{array}{l}\text { CA/CE/MA/ } \\
\text { PP/PT }\end{array}$ \\
\hline Fabaceae & Sesbania virgata (Cav.) Pers. & 15410 & AV & IV & EE & $\begin{array}{l}\text { CA/CE/MA/ } \\
\text { PP/PT }\end{array}$ \\
\hline Fabaceae & Stylosanthes guianensis (Aubl.) Sw. & 9311 & ER & VI & NE & $\begin{array}{l}\text { AM/CA/CE/ } \\
\text { MA/PP/PT }\end{array}$ \\
\hline Fabaceae & Stylosanthes viscosa Sw. & 8583 & ER & VNI & NE & $\mathrm{AM} / \mathrm{CA} / \mathrm{CE} / \mathrm{MA}$ \\
\hline Fabaceae & Zornia reticulata Sm. & 15413 & ER & VNI & NE & $\begin{array}{l}\text { AM/CA/CE/ } \\
\text { MA/PP/PT }\end{array}$ \\
\hline Gentianaceae & $\begin{array}{c}\text { Calolisianthus speciosus (Cham. \& } \\
\text { Schltdl.) Gilg. }\end{array}$ & 8566 & ER & VI & $\mathrm{EE}$ & CE \\
\hline
\end{tabular}




\begin{tabular}{|c|c|c|c|c|c|c|}
\hline Gentianaceae & Schultesia guianensis (Aubl.) Malme & 11014 & ER & VI & NE & $\mathrm{AM} / \mathrm{CA} / \mathrm{CE} / \mathrm{MA}$ \\
\hline Heliconiaceae & Heliconia psittacorum L.f. & 8565 & ER & $\begin{array}{l}\text { FI/IV/VI/ } \\
\text { VNI }\end{array}$ & $\mathrm{NE}$ & $\begin{array}{l}\text { AM/CA/CE/ } \\
\text { MA/PT }\end{array}$ \\
\hline Humiriaceae & Humiria balsamifera (Aubl.) J.St.-Hil. & 9332 & $A B$ & IV & $\mathrm{NE}$ & $\mathrm{AM} / \mathrm{CA} / \mathrm{CE} / \mathrm{MA}$ \\
\hline Humiriaceae & Sacoglottis mattogrossensis Malme & 11320 & AV & IV & NE & AM/CE/MA \\
\hline Hypericaceae & Vismia guianensis (Aulb.) Choisy. & 9336 & $A B$ & IV & NE & $\mathrm{AM} / \mathrm{CA} / \mathrm{CE} / \mathrm{MA}$ \\
\hline Hypoxidaceae & $\begin{array}{c}\text { Curculigo scorzonerifolia (Lam.) } \\
\text { Baker }\end{array}$ & 11482 & ER & VNI & $\begin{array}{c}\text { Des- } \\
\text { co- } \\
\text { nh. }\end{array}$ & $\mathrm{AM} / \mathrm{CE} / \mathrm{MA}$ \\
\hline Iridaceae & Trimezia martinicensis (Jacq.) Herb. & 15716 & ER & VI & $\mathrm{NE}$ & $\mathrm{AM} / \mathrm{CA} / \mathrm{CE} / \mathrm{MA}$ \\
\hline Lamiaceae & Hyptis lantanifolia Poit. & 8576 & ER & VI & NE & $\mathrm{AM} / \mathrm{CA} / \mathrm{CE} / \mathrm{MA}$ \\
\hline Lamiaceae & $\begin{array}{c}\text { Marsypianthes chamaedrys (Vahl) } \\
\text { Kuntze }\end{array}$ & 10999 & $\mathrm{ER}$ & VI & NE & $\begin{array}{l}\text { AM/CA/CE/ } \\
\text { MA/PT }\end{array}$ \\
\hline Lauraceae & Cassytha filiformis $\mathrm{L}$. & 7864 & LI & $\begin{array}{l}\mathrm{FI} / \mathrm{IV} / \mathrm{VI} / \\
\text { VNI }\end{array}$ & $\mathrm{NE}$ & $\mathrm{AM} / \mathrm{CA} / \mathrm{CE} / \mathrm{MA}$ \\
\hline Lauraceae & Ocotea lobbii (Meisn.) Rohwer & 9305 & $A B$ & IV & EE & MA \\
\hline Lecythidaceae & $\begin{array}{c}\text { Eschweilera ovata (Cambess.) Mart. } \\
\text { ex Miers }\end{array}$ & 7888 & AV & FI & $\mathrm{EE}$ & AM/MA \\
\hline Lentibulariaceae & Genlisea filiformis A.St.-Hil & 7885 & ER & VI & $\mathrm{NE}$ & $\mathrm{AM} / \mathrm{CA} / \mathrm{CE}$ \\
\hline Lentibulariaceae & Utricularia subulata L. & 11008 & ER & VI & NE & $\mathrm{AM} / \mathrm{CA} / \mathrm{CE} / \mathrm{MA}$ \\
\hline Loranthaceae & Psittacanthus dichroos (Mart.) Mart. & 11293 & ER & & EE & $\mathrm{AM} / \mathrm{CA} / \mathrm{CE} / \mathrm{MA}$ \\
\hline Loranthaceae & Psittacanthus robustus (Mart.) Mart. & 7910 & ER & IV & NE & $\begin{array}{l}\text { AM/CA/CE/ } \\
\text { MA/PT }\end{array}$ \\
\hline Loranthaceae & $\begin{array}{c}\text { Struthantus marginatus (Desr.) } \\
\text { Blume }\end{array}$ & 11315 & ER & IV & NE & $\mathrm{AM} / \mathrm{CA} / \mathrm{CE} / \mathrm{MA}$ \\
\hline Loranthaceae & Struthanthus polyrhizus (Mart.) Mart. & 15405 & $\mathrm{ER}$ & IV & $\mathrm{NE}$ & $\mathrm{CA} / \mathrm{CE} / \mathrm{MA}$ \\
\hline Lythraceae & Cuphea antisyphilitoca Kunth. & 9329 & ER & VI & $\mathrm{NE}$ & AM/CE/MA \\
\hline Lythraceae & Cuphea brachiata Koehne & 7867 & ER & VI & EE & $\mathrm{CA} / \mathrm{MA}$ \\
\hline Lythraceae & Cuphea sessiliflora A.ST-Hil & 7884 & ER & VI & EE & CE/MA \\
\hline Lythraceae & Pleurophora anomala Koehne & 11024 & ER & VI & EE & $\mathrm{CA} / \mathrm{CE}$ \\
\hline Malpighiaceae & Byrsonima dealbata Griseb. & 8577 & $A B$ & IV/VNI & EE & $\mathrm{CA} / \mathrm{CE}$ \\
\hline Malpighiaceae & $\begin{array}{l}\text { Niedenzuella multiglandulosa } \\
\text { (A.Juss.) W.R.Anderson }\end{array}$ & 8579 & LI & IV & $\mathrm{EE}$ & AM/CE/MA/PT \\
\hline Malpighiaceae & Stigmaphyllon paralias A. Juss & 15062 & ER & VI/VNI & $\mathrm{EE}$ & $\mathrm{CA} / \mathrm{CE} / \mathrm{MA}$ \\
\hline Malvaceae & Hibiscus bifurcatus Cav. & 15408 & $A B$ & VI & $\mathrm{NE}$ & AM/MA \\
\hline Malvaceae & $\begin{array}{l}\text { Peltaea obsita (Mart. ex Colla) } \\
\text { Krapov. \& Cristóbal }\end{array}$ & 7870 & $A B$ & VI & NE & $\mathrm{CE} / \mathrm{MA} / \mathrm{PT}$ \\
\hline
\end{tabular}




\begin{tabular}{|c|c|c|c|c|c|c|}
\hline Malvaceae & Sida linifolia Cav. & 11310 & ER & VI & NE & $\begin{array}{l}\text { AM/CA/CE/ } \\
\text { MA/PT }\end{array}$ \\
\hline Malvaceae & Sida ciliares L. & 9348 & ER & VI & $\mathrm{DE}$ & $\mathrm{CA} / \mathrm{MA}$ \\
\hline Malvaceae & Triumfetta semitriloba Jacq. & 7907 & $A B$ & VI & NE & AM/CA/CE/MA \\
\hline Malvaceae & Waltheria cinerescens A.St-Hil. & 15078 & $A B$ & VNI & EE & $\mathrm{CA} / \mathrm{CE}$ \\
\hline Marantaceae & $\begin{array}{c}\text { Monotagma plurispicatum (Körn.) } \\
\text { K.Schum. }\end{array}$ & 15308 & ER & FI & $\mathrm{NE}$ & $\begin{array}{l}\text { AM/CA/CE/ } \\
\text { MA/PT }\end{array}$ \\
\hline Marantaceae & Goeppertia effusa Saka \& Lombardi & 15420 & ER & FI & EE & $\mathrm{CA} / \mathrm{CE} / \mathrm{MA}$ \\
\hline Mayacaceae & Mayaca fluviatilis Aubl. & 15716 & ER & VI & NE & $\begin{array}{l}\text { AM/CA/CE/ } \\
\text { MA/PT }\end{array}$ \\
\hline $\begin{array}{l}\text { Melastomata- } \\
\text { ceae }\end{array}$ & Clidemia capitellata (L.) D.Don & 7862 & $A B$ & FI/IV & $\mathrm{NE}$ & $\mathrm{AM} / \mathrm{CA} / \mathrm{CE} / \mathrm{MA}$ \\
\hline $\begin{array}{l}\text { Melastomata- } \\
\text { ceae }\end{array}$ & Clidemia hirta (L.) D.Don & 9309 & $A B$ & $\mathrm{FI} / \mathrm{IV}$ & NE & $\mathrm{AM} / \mathrm{CA} / \mathrm{CE} / \mathrm{MA}$ \\
\hline $\begin{array}{l}\text { Melastomata- } \\
\text { ceae }\end{array}$ & Comolia ovalifolia (DC.) Triana & 7880 & ER & VI & EE & MA \\
\hline $\begin{array}{l}\text { Melastomata- } \\
\text { ceae }\end{array}$ & Marcetia taxifolia (A.St-Hil.)Dc. & 8575 & $A B$ & VI & $\mathrm{EE}$ & $\mathrm{AM} / \mathrm{CA} / \mathrm{CE} / \mathrm{MA}$ \\
\hline $\begin{array}{l}\text { Melastomata- } \\
\text { ceae }\end{array}$ & Miconia ciliata (Ferreira.C.A) & 7889 & $A B$ & VI & $\mathrm{NE}$ & $\mathrm{AM} / \mathrm{CA} / \mathrm{CE} / \mathrm{MA}$ \\
\hline $\begin{array}{l}\text { Melastomata- } \\
\text { ceae }\end{array}$ & Miconia Iurida Cogn. & 7887 & AV & FI & EE & MA \\
\hline $\begin{array}{l}\text { Melastomata- } \\
\text { ceae }\end{array}$ & Miconia holosericea (L.) DC. & 11005 & AV & FI & NE & AM/CE/MA \\
\hline $\begin{array}{l}\text { Melastomata- } \\
\text { ceae }\end{array}$ & Microlicia sp. & 8569 & ER & VI & 0 & \\
\hline $\begin{array}{l}\text { Melastomata- } \\
\text { ceae }\end{array}$ & $\begin{array}{c}\text { Pterolepis cataphracta (Cham.) } \\
\text { Triana }\end{array}$ & 7869 & $A B$ & VI & $\mathrm{EE}$ & $\mathrm{CA} / \mathrm{CE}$ \\
\hline $\begin{array}{l}\text { Melastomata- } \\
\text { ceae }\end{array}$ & Pterolepis glomerata (Rottb.) Miq. & 11020 & ER & VI & EE & $\mathrm{AM} / \mathrm{CA} / \mathrm{CE} / \mathrm{MA}$ \\
\hline $\begin{array}{l}\text { Melastomata- } \\
\text { ceae }\end{array}$ & $\begin{array}{c}\text { Pleroma urceolaris (Schrank et Mart. } \\
\text { ex DC.) Triana }\end{array}$ & 15076 & $A B$ & IV & EE & MA \\
\hline Metteniusaceae & Emmotum affine Miers & - & $A B$ & IV & EE & MA \\
\hline Myrtaceae & Eugenia astringens Cambess & 15724 & AV & VNI & EE & MA \\
\hline Myrtaceae & Myrcia guianensis (Aubl.) DC. & 15417 & AV & IV & $\mathrm{NE}$ & $\mathrm{AM} / \mathrm{CA} / \mathrm{CE} / \mathrm{MA}$ \\
\hline Myrtaceae & Myrcia salzmanii O.Berg. & 15067 & $A B$ & IV & EE & MA \\
\hline Myrtaceae & Myrcia hirtiflora DC. & 11022 & $A B$ & IV & $\mathrm{EE}$ & MA \\
\hline Myrtaceae & Myrcia spathulifolia Proença & 15061 & $A B$ & IV & $\mathrm{EE}$ & MA \\
\hline Nyctaginaceae & $\begin{array}{c}\text { Guapira pernambucensis (Casar.) } \\
\text { Lundell }\end{array}$ & 9316 & $A B$ & $\mathrm{FI} / \mathrm{IV}$ & EE & MA \\
\hline Ochnaceae & Ouratea gigantophylla (Erhard) Engl. & 11314 & $A B$ & $\mathrm{FI}$ & EE & MA \\
\hline Ochnaceae & Ouratea suaveolens (A.St.-Hil.) Engl. & 11312 & $A B$ & IV/VI & $\mathrm{EE}$ & CE/MA \\
\hline Ochnaceae & Sauvagesia erecta L. & 9321 & ER & VI & $\mathrm{NE}$ & AM/CA/CE/MA \\
\hline
\end{tabular}




\begin{tabular}{|c|c|c|c|c|c|c|}
\hline Ochnaceae & Sauvagesia longifolia Eichler & 8573 & ER & VI & $\mathrm{NE}$ & AM/MA \\
\hline Ochnaceae & Sauvagesia sprengelii A.St.-Hil & 8574 & ER & VI & $\mathrm{NE}$ & AM/MA \\
\hline Onagraceae & $\begin{array}{c}\text { Ludwigia octovalvis (Jacq.) P.H. } \\
\text { Raven }\end{array}$ & 10994 & $A B$ & VI & $\mathrm{NE}$ & $\begin{array}{l}\text { AM/CA/CE/ } \\
\text { MA/PT }\end{array}$ \\
\hline Orchidaceae & Epidendrum cinnabarinum Salzm. & 11287 & ER & IV/VNI & EE & CA/CE/MA \\
\hline Orchidaceae & $\begin{array}{c}\text { Epidendrum orchidiflorum (Salzm.) } \\
\text { Lindl. }\end{array}$ & 9324 & ER & IV/VNI & EE & $\mathrm{AM} / \mathrm{CA} / \mathrm{CE} / \mathrm{MA}$ \\
\hline Orchidaceae & Epistephium williamsii Hook.f. & 9330 & ER & VI & $\mathrm{EE}$ & CE \\
\hline Orchidaceae & Pelexia sp. & 11013 & ER & VI & 0 & \\
\hline Orchidaceae & Vanilla bahiana Hoehne & 9338 & LI & IV/VNI & EE & $\mathrm{CA} / \mathrm{CE} / \mathrm{MA}$ \\
\hline Orobanchaceae & Esterhazya splendida J.C.Mikan & 8572 & $A B$ & VI & NE & CA/CE/MA \\
\hline Passifloraceae & Passiflora contracta Vitta & 11019 & $\mathrm{LI}$ & IV & EE & $\mathrm{CA} / \mathrm{MA}$ \\
\hline Passifloraceae & Passiflora silvestris Vell. & 15404 & LI & IV & EE & $\mathrm{CE} / \mathrm{MA}$ \\
\hline Peraceae & $\begin{array}{c}\text { Chaetocarpus echinocarpus (Baill.) } \\
\text { Ducke }\end{array}$ & 11286 & $A B$ & IV & NE & $\mathrm{AM} / \mathrm{CA} / \mathrm{CE}$ \\
\hline Peraceae & $\begin{array}{c}\text { Pera glabrata (Schott.) Poepp. Ex } \\
\text { Baill }\end{array}$ & 11017 & $A B$ & IV & NE & $\mathrm{AM} / \mathrm{CA} / \mathrm{CE} / \mathrm{MA}$ \\
\hline Phyllanthaceae & Phyllanthus klotzschianus Müll.Arg. & 9306 & ER & VNI & EE & CE/MA \\
\hline Plantaginaceae & $\begin{array}{c}\text { Achetaria ocymoides (Cham. \& Schl- } \\
\text { tdl.) Wettst. }\end{array}$ & 15314 & ER & VI & EN & $\mathrm{CA} / \mathrm{CE} / \mathrm{MA}$ \\
\hline Plantaginaceae & Stemodia foliosa Benth. & 11007 & $A B$ & VI & $\mathrm{NE}$ & CA/CE/MA \\
\hline Poaceae & Axonopus aureus P. Beauv. & 9345 & ER & VNI & NE & $\mathrm{AM} / \mathrm{CA} / \mathrm{CE} / \mathrm{MA}$ \\
\hline Poaceae & Echinolaena inflexa (Poir.) Chase & 9317 & ER & VNI & NE & $\mathrm{AM} / \mathrm{CA} / \mathrm{CE} / \mathrm{MA}$ \\
\hline Poaceae & $\begin{array}{c}\text { Dichanthelium hebotes (Trin.) Zu- } \\
\text { loaga }\end{array}$ & 15403 & ER & VI & NE & CE/MA \\
\hline Polygalaceae & $\begin{array}{c}\text { Asemeia ovata (Poir.) J.F.B.Pastore \& } \\
\text { J.R.Abbott }\end{array}$ & 15040 & ER & VNI & NE & $\begin{array}{l}\text { AM/CA/CE/ } \\
\text { MA/PT }\end{array}$ \\
\hline Polygalaceae & Polygala paniculata L. & 15064 & ER & VNI & NE & $\begin{array}{l}\text { AM/CA/CE/ } \\
\text { MA/PP }\end{array}$ \\
\hline Polygalaceae & Polygala timoutou Aubl. & 9300 & ER & VNI & $\mathrm{EE}$ & AM/CE/MA \\
\hline Polygalaceae & Polygala trichosperma Jacq. & 15064 & ER & VNI & NE & $\mathrm{AM} / \mathrm{CA} / \mathrm{CE} / \mathrm{MA}$ \\
\hline Polygonaceae & Coccoloba rosea Meisn. & 10982 & $A B$ & FI/IV & EE & MA \\
\hline Polygonaceae & Coccoloba laevis Casar. & 15042 & $A B$ & FI/IV & EE & MA \\
\hline Blechnaceae & $\begin{array}{l}\text { Telmatoblechnum serrulatum (Rich.) } \\
\text { Perrie, D.J. Ohlsen \& Brownsey }\end{array}$ & 11316 & ER & VI & NE & $\mathrm{AM} / \mathrm{CE} / \mathrm{MA}$ \\
\hline Rubiaceae & Pagamea guianensis Aubl. & 11313 & $A B$ & IV & NE & AM/CA/CE/MA \\
\hline
\end{tabular}




\begin{tabular}{|c|c|c|c|c|c|c|}
\hline Rubiaceae & Amaioua guianensis Aubl. & 11318 & $A B$ & IV & NE & $\mathrm{AM} / \mathrm{CE} / \mathrm{MA}$ \\
\hline Rubiaceae & Guettarda platypoda DC. & 15043 & $A B$ & FI & EE & AM/MA \\
\hline Rubiaceae & Cordiera obtusa (K.Schum.) Kuntze & 15407 & $A B$ & IV & $\mathrm{EE}$ & CE \\
\hline Rubiaceae & Borreria cf. verticillata (L.) G.Mey. & 10979 & ER & VI/VNI & NE & $\mathrm{AM} / \mathrm{CA} / \mathrm{CE} / \mathrm{MA}$ \\
\hline Rubiaceae & $\begin{array}{l}\text { Denscantia cymosa (Spreng.) } \\
\text { E.L.Cabral \& Bacigalupo }\end{array}$ & 10997 & LI & IV & $\mathrm{EE}$ & MA \\
\hline Rubiaceae & $\begin{array}{c}\text { Denscantia monodon (K.Schum.) } \\
\text { E.L.Cabral \& Bacigalupo }\end{array}$ & 15086 & $\mathrm{LI}$ & IV & $\mathrm{EE}$ & MA \\
\hline Rubiaceae & $\begin{array}{l}\text { Hexasepalum apiculatum (Willd.) } \\
\text { Delprete \& J.H. Kirkbr. }\end{array}$ & 11015 & ER & VI & NE & $\mathrm{AM} / \mathrm{CA} / \mathrm{CE} / \mathrm{PP}$ \\
\hline Rubiaceae & Chiococca plowmanii Delprete & 15083 & $A B$ & FI/IV & $\mathrm{EE}$ & MA \\
\hline Rubiaceae & Chiococca alba (L.) Hitchc. & 15068 & $A B$ & FI/IV & NE & $\begin{array}{l}\text { AM/CA/CE/ } \\
\text { MA/PT }\end{array}$ \\
\hline Rubiaceae & $\begin{array}{c}\text { Emmeorhiza umbellata (Spreng.) } \\
\text { K.Schum. }\end{array}$ & 11001 & LI & IV & NE & $\begin{array}{l}\text { AM/CA/CE/ } \\
\text { MA/PT }\end{array}$ \\
\hline Rubiaceae & $\begin{array}{c}\text { Pentodon pentandrus (Schumach. \& } \\
\text { Thonn.) Vatke*** }\end{array}$ & 15306 & ER & VI & NE & $\mathrm{AM} / \mathrm{CE} / \mathrm{MA}$ \\
\hline Rubiaceae & Perama hirsuta Aubl. & 11010 & ER & VI & NE & $\mathrm{AM} / \mathrm{CA} / \mathrm{CE} / \mathrm{MA}$ \\
\hline Rubiaceae & Posoqueria latifolia (Rudge) Schult. & 15307 & AV & FI & NE & $\begin{array}{l}\text { AM/CA/CE/ } \\
\text { MA/PM/PT }\end{array}$ \\
\hline Rubiaceae & Psychotria bahiensis DC. & 8578 & $A B$ & FI/IV & NE & $\mathrm{AM} / \mathrm{CA} / \mathrm{CE} / \mathrm{MA}$ \\
\hline Rubiaceae & Psychotria jambosioides Schltdl. & 15415 & $A B$ & FI/IV & EE & MA \\
\hline Rubiaceae & Sabicea grisea Cham. \& Schltall. & 9319 & $\mathrm{LI}$ & IV & NE & $\mathrm{AM} / \mathrm{CE} / \mathrm{MA}$ \\
\hline Rubiaceae & Salzmannia nitida DC. & 8580 & $A B$ & IV & $\mathrm{EE}$ & MA \\
\hline Rubiaceae & $\begin{array}{l}\text { Staelia cf. virgata (Link ex Roem. \& } \\
\text { Schult.) K.Schum. }\end{array}$ & 9303 & ER & VI & NE & $\begin{array}{l}\text { AM/CA/CE/ } \\
\text { MA/PT }\end{array}$ \\
\hline Sapindaceae & Urvillea laevis Radlk. & 7914 & $\mathrm{LI}$ & IV & NE & $\mathrm{CA} / \mathrm{CE} / \mathrm{MA} / \mathrm{PT}$ \\
\hline Smilacaceae & Smilax staminea Griseb & 9322 & $\mathrm{LI}$ & IV & NE & CE/MA \\
\hline Urticaceae & Cecropia pachystachia Trécul & 10984 & AV & FI & NE & $\begin{array}{l}\text { AM/CA/CE/ } \\
\text { MA/PT }\end{array}$ \\
\hline Velloziaceae & Vellozia dasypus Seub. & 7908 & ER & VNI & $\mathrm{EE}$ & $\mathrm{CA} / \mathrm{CE} / \mathrm{MA}$ \\
\hline Verbenaceae & Lantana fucata Lindl. & 15069 & $A B$ & VNI & NE & $\mathrm{CA} / \mathrm{CE} / \mathrm{MA}$ \\
\hline Verbenaceae & Lantana salzmannii Schauer & 10998 & $A B$ & VNI & EE & MA \\
\hline Verbenaceae & Lantana Iucida Schauer & 15418 & $A B$ & VNI & $\mathrm{EE}$ & MA \\
\hline Verbenaceae & Tamonea spicata Aubl. & 9342 & ER & VI & NE & $\mathrm{CA} / \mathrm{CE} / \mathrm{MA}$ \\
\hline Verbenaceae & Stachytarpheta lactea Schaumer & 15715 & ER & VI & $\mathrm{EE}$ & $\mathrm{CA} / \mathrm{CE} / \mathrm{MA}$ \\
\hline
\end{tabular}


TAB. 2 - CONTINUAÇÃO

\begin{tabular}{cccccccc}
\hline Vitaceae & Cissus erosa Rich & 15719 & LI & VNI & EE & AM/CA/CE/MA \\
Vochysiaceae & Vochysia lucida C. Presl. & 11295 & AB & FI & EE & MA \\
Xyridaceae & Xyris ciliata Thunb. & 11002 & ER & VI & EE & CE \\
Xyridaceae & Xyris sp. & 9320 & ER & VI & & \\
Xyridaceae & Xyris jupicai Rich. & 15066 & ER & VI & NE & AM/CA/CE/ \\
\hline
\end{tabular}

Mais de $50 \%$ das espécies da área não são endêmicas do Brasil (Fig. 3). Quando se avalia a distribuição por Domínios Fitogeográficos, apenas 33 espécies são exclusivas do Domínio Fitogeográfico da Mata Atlântica, enquanto nove espécies ocorrem nos seis domínios fitogeográficos brasileiros (Tab. 1). Onze espécies são comuns apenas entre o Domínio Fitogeográfico da Amazônia e Domínio Fitogeográfico da Floresta Atlântica (DFFA), enquanto oito são comuns entre o Domínio Fitogeográfico da Caatinga e o DFFA, sete entre o Domínio Fitogeográfico do Cerrado e o DFFA. Vinte espécies não eram apontadas no DFFA.

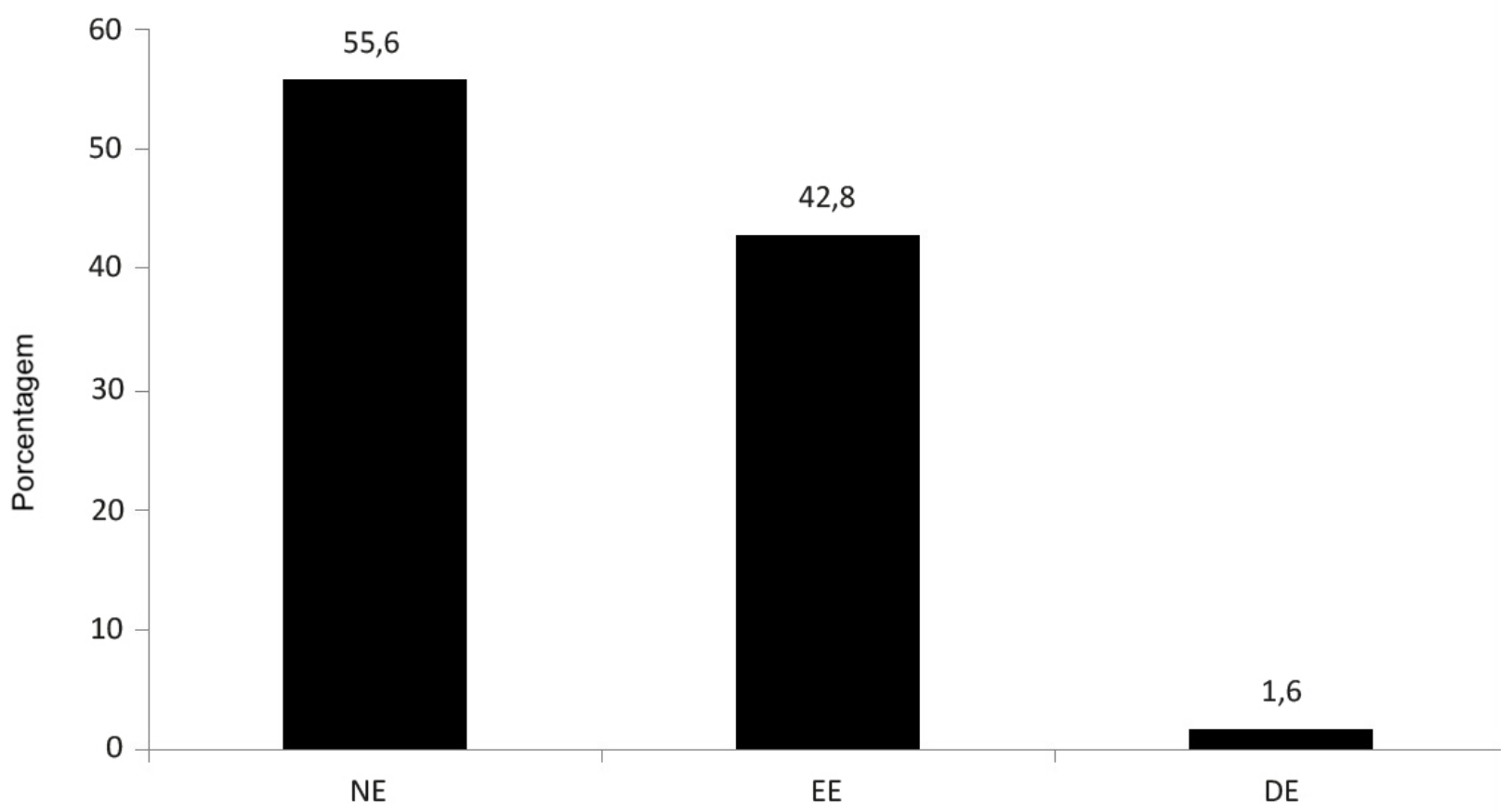

Fig. 3. Porcentagem de hábitos encontrados em restinga, Jaguaripe, Bahia, Brasil. NE - não endêmica do Brasil; EE - é endêmica do Brasil; DE - desconhecido.

Ao se observar o habitat das espécies na restinga estudada, 28 espécies ocorreram na FI, com 50\% dessas espécies exclusivas desse habitat (e.g. Albizia polycephala (Benth.) Killip ex Record e Vochysia lucida C. Presl.). 54 espécies ocorreram na IV, das quais $72,2 \%$ foram exclusivas (e.g. Sacoglottis mattogrossensis Malme e Vriesea neoglutinosa Mez). Na VI, ocorreram 77 espécies com 88,3\% exclusivas (e.g. Genlisea filiformis A.St.-Hil. e Paepalanthus bifidus (Schrad.) Kunth ), enquanto na VNI ocorreram 60 espécies com 76,6\% são exclusivas (e.g. Aechmea mertensii (G. Mey.) Schult. \& Schult.f. e Vellozia dasypus Seub.). Somente Heliconia psittacorum L.f. e Cassytha filiformis L. ocorreram em todos os habitats amostrados.

\section{DIscussão}

O número de 195 espécies encontradas no município de Jaguaripe está entre os menores valores de riqueza de espécies encontrados para as restingas, que variam entre 154 e 343 (Quei- 


\section{ANÁLISE DE SIMILARIDADE}

A compilação dos estudos florísticos resultou em uma lista de 1305 espécies, após a exclusão das únicas ocorrências o total de 507 espécies foi considerado na análise. A análise de Cluster obteve um coeficiente de correlação cofenética de 0,96. Foram evidenciados grupos relacionados com baixa similaridade (Fig. 4). Dois grupos são reconhecidos a $12 \%$ de similaridade, o primeiro formado apenas por restingas da região sudeste e o segundo por restingas localizadas na região nordeste. Considerando $50 \%$ de similaridade, apenas um grupo é evidenciado formado por duas áreas de restinga localizadas na Bahia.

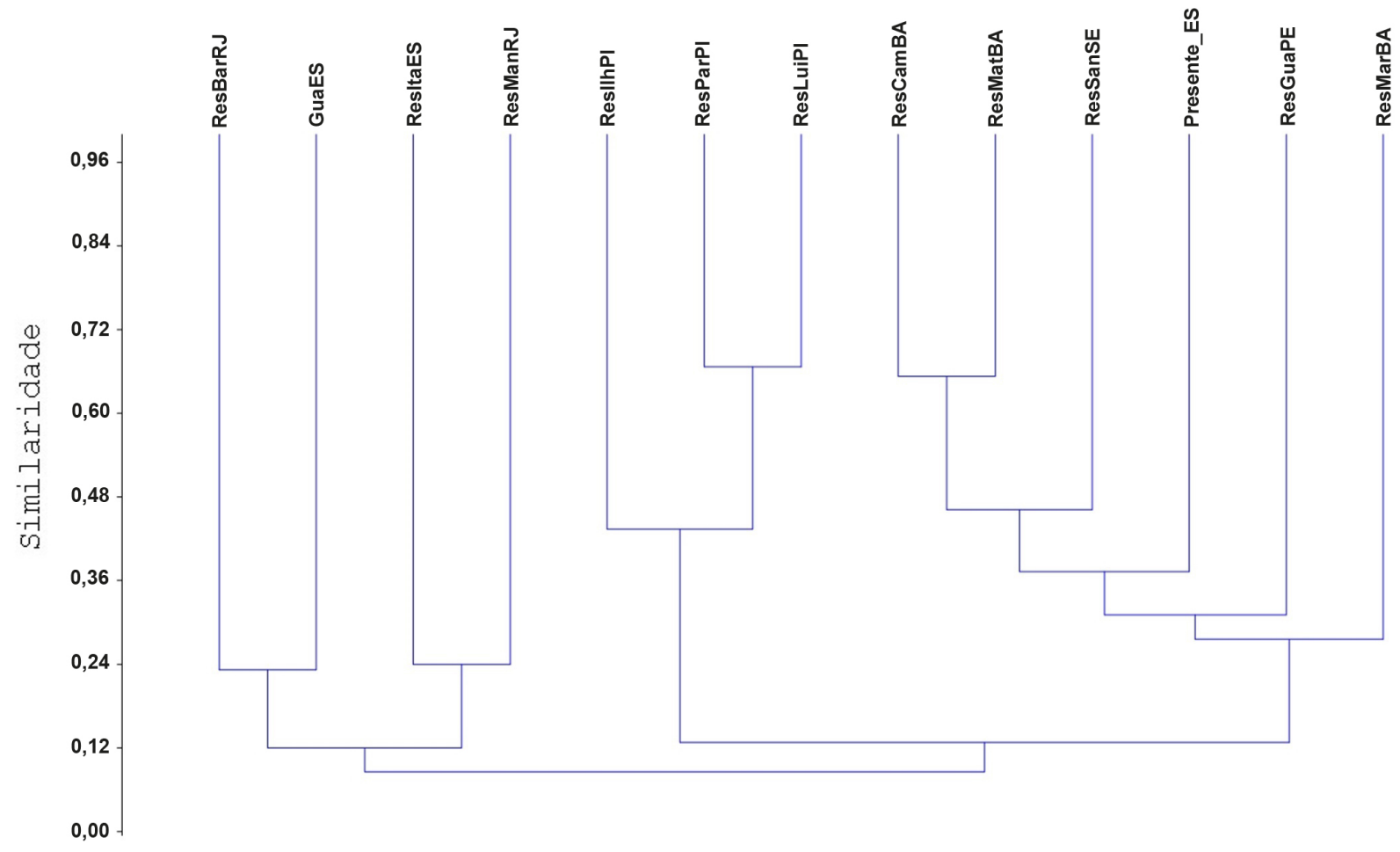

Fig. 4. Análise de cluster, utilizando o Sorensen como índice de similaridade em 15 áreas de restinga no Brasil.

roz et al., 2012; Fernandes \& Queiroz, 2015) e estudos realizados pela costa brasileira dentro desse intervalo (Pereira et al., 1992; Pereira \& Araújo, 1995; Fabris \& Pereira, 1998; Araujo et al., 2004; Rocha et al., 2007; Colodete \& Pereira, 2007; Magnago et al., 2011b).

Estudos florísticos em outras restingas da Bahia têm apontado a ocorrência das mesmas famílias encontradas neste estudo como as de maior riqueza em espécies (e.g. Queiroz et al., 2012; Fernandes \& Queiroz et al., 2015), bem como em outras áreas de restinga da costa brasileira (e.g. Castelo \& Braga, 2017).

Myrtaceae normalmente é referenciada como predominante em riqueza e abundância nas restingas (Souza et al., 2007; Menezes et al., 2012; Lourenço \& Barbosa, 2012). Entretanto, a restinga de Jaguaripe não apresentou elevada diversidade de Myrtaceae. Áreas de restinga arbustiva tendem a concentrar poucas espécies de Myrtaceae, entre 1 a 9 de espécies (MeiraNeto et al., 2005; Monteiro et al., 2014; Castelo \& Braga, 2017). Em contrapartida, restingas com formações florestais, principalmente não inundáveis (formação não ocorrente na restinga estudada), concentram um grande número de espécies de Myrtaceae, variando entre 16 e 32 espécies (Assis et al., 2004; Martins et al., 2008; Moraes \& Magenta, 2014).

Entre as famílias com maior riqueza em espécies (Tabela 2), Rubiaceae apresenta cinco endêmicas do domínio fitogeográfico da Floresta Atlântica (Roque \& Carvalho 2011; BFG 2015). Já entre as Fabaceae, apenas duas são endêmicas desse domínio. Restingas são ecossistemas de origem recente e por isso, o número de espécies endêmicas e/ou linhagens exclusivas é reduzido (Scarano, 2002). O compartilhamento de espécies entre Floresta Atlântica/Amazônia 
acompanha a disjunção proposta para os dois domínios, possivelmente evidenciada pelas flutuações climáticas do quaternário (Fiaschi \& Pirani, 2009).

A maior quantidade de espécies do componente herbáceo, seguido pelo arbustivo, segue o predomínio de formações herbáceas e arbustivas e a ocorrência limitada de formação florestal. Além disso, florestas inundadas e inundáveis tendem a apresentar menor riqueza quando comparadas a outras formações vegetais de restinga (Magnago et al., 2011b).

Foram registradas espécies endêmicas das restingas e uma espécie ameaçada de extinção e distribuição geográfica, como é o caso de Aechmea blanchetiana (Baker) L.B.Sm, espécie quase ameaçada (BFG, 2015), com ocorrência na Bahia e Espírito Santo.

Algumas espécies encontradas em Jaguaripe são endêmicas das restingas do Nordeste do Brasil, e.g. Calea angusta S.F.Blake (BA e SE), Clusia sellowiana Schltdl. (Al, BA e SE) e Salzmannia nitida DC. (AL, BA, PE, PB, RN e SE) (BFG, 2015). Outras espécies ocorrentes na área de estudo são endêmicas das restingas, com ocorrência em estados das regiões nordeste e sudeste como Ilex psammophila Reissek (BA e ES) e Pterolepis cataphracta (Cham.) Triana (BA, ES e SE), ressaltando a importância desse tipo de estudo para reconhecimento de padrões florísticos (BFG, 2015).

A restinga de Jaguaripe é aqui reconhecida como refúgio para espécies endêmicas da Bahia, incluindo espécies nunca antes relacionadas para este tipo vegetacional como: Ouratea gigantophylla (Erhard) Engl., endêmica de florestas estacionais deciduais (BFG 2015). Vochysia lucida C.Presl com ocorrência relatada para matas de galeria ou ciliares (BFG 2015). Miconia lurida Cogn. referida apenas como ocorrente em florestas ombrófilas, sendo registrada pela primeira vez para restinga, ampliando sua área de distribuição. Guatteria ferruginea A.St.-Hill, apesar de não ser endêmica do estado da Bahia, nunca tinha sido registrada a ocorrência em formações de restinga (BFG, 2015).

Espécies como Actinocephalus herzogii var. humilis (Sano) Sano, Bonnetia stricta (Nees) Nees \& Mart., Byrsonima dealbata Griseb., Cuphea brachiate Mart. ex Koehne, Phyllanthus klotzschianus Müll. Arg. e Vellozia dasypus Seub. reforçam a bem conhecida disjunção entre campo rupestre e as restingas (Harley 1995, Costa et al. 2010). Na revisão da disjunção proposta por Alves et al. (2007), somente $B$. stricta e $V$. dasypus constam nas espécies citadas na disjunção.
O estudo registrou quatro espécies naturalizadas na restinga de Jaguaripe: Crotalaria retusa L., Desmodium adscendens (Sw.) DC., Desmodium triflorum (L.) DC. e Pentodon pentandrus (Schumach. \& Thonn.) Vatke que ocorrem nas formações arbustivas não-inundáveis. Casuarina equisetifolia L. é de origem australiana, invasora de ambientes costeiros (Rodgers III \& Ambinakudige, 2012), com dispersão anemocórica e produção de numerosas sementes, seguindo um padrão fenológico de frutificação com tendência infraanual (Hammerton, 2001; Swearingen, 2008). No estudo, esse foi um fator de maior preocupação, devido à ocorrência de diversos indivíduos jovens de $C$. equisetifolia verificados próximos à linha de praia, o que indica recrutamento e estabelecimento dessa espécie. As restingas são alvo de forte antropização e essa característica se reflete na flora da restinga de Jaguaripe pela presença desses diversos elementos alóctones, reforçando o estado de alerta para esse tipo de vegetação na costa do Brasil.

A formação de grupos com baixa similaridade pode ser influenciada, provavelmente, pelos diversos fatores que interferem no estabelecimento da vegetação de restinga (Araújo, 2000; Magnago et al., 2010; SaporettiJunior et al., 2012). A distinção entre as restingas do nordeste e sudeste no tocante à similaridade florística já foram evidenciadas para as formações florestais (Fernandes \& Queiroz 2015), padrão aqui reforçado com a amostragem de distintas fisionomias. Em complemento a essa ideia, abordagens biogeográficas com espécies de Kielmeyera Mart. \& Zucc. ocorrentes na restinga, representada no presente estudo por Kielmeyera neglecta Saddi [espécie distribuída em restinga e borda de florestas ombrófilas (Santos et al., 2015)], indicaram a bacia do Rio Doce como área de confluência deste gênero, apontando dissimilaridade em relação ao norte e sul deste rio (Santos et al., 2016).

Os dados levantados a partir do inventário florístico na restinga de Jaguaripe reforçam a importância de conhecer as espécies nos ambientes, ao apresentar dados sobre endemismos, novas ocorrências, espécies exóticas e invasoras, além das suas distribuições em distintas fisionomias. Esses dados podem se constituir em ferramentas úteis às políticas de conservação ao sinalizar áreas prioritárias, além de contribuir ao conhecimento em geral da flora das restingas. 


\section{Agradecimentos}

Os autores agradecem aos seguintes taxonomistas pela ajuda na identificação: A. Amorim (Malpighiaceae); A.K.A. Santos Melastomataceae); A.P.B. Santos (Calophyllaceae); A. Rapini Apocynaceae); A. Stadnick (Myrtaceae); C.A. Ferreira Melastomataceae); C. Pessoa (Malpighiaceae); C.van den Berg Orchidaceae); D. Cardoso (Fabaceae); D.N. Carvalho Orchidaceae); F.M. Guedes (Lentibulariaceae); F.S. Souza Nyctaginaceae); G. V. Fonseca (Asteraceae); G. Siqueira Marantaceae); L. Marinho (Hypericaceae); L. Queiroz Fabaceae); L. Versiaux (Bromeliaceae); J.D.O. Melo Myrtaceae); J. Jardim Rubiaceae); L.M. Brauner (Lythraceae); M. Alves(Asteraceae); M. Cota (Fabaceae); M.G. Facco Lythraceae); M.L. Guedes Aquifoliaceae, Asteraceae); M. Trovó (Eriocaulaceae); N. Roque Asteraceae); N. Souza (Onagraceae); P.L. Moraes (Lauraceae); P.L. Ribeiro Orchidaceae); R. Goldenberg (Melastomataceae); T.S. Nunes (Passifloraceae); W.O. Fonseca (Rubiaceae). Agradecemos ao H.C.A. França por apresentar a região de Jaguaripe; ao L.C Marinho pela confecção da prancha e mapa. LYSA agradece à Fundação de Amparo à Pesquisa do Estado da Bahia FAPESB) e ao Conselho Nacional de Desenvolvimento Científico e Tecnológico (CNPq), pelo financiamento de projetos de pesquisa (Processos APP0113/2009 e 482085/2009-6, respectivamente).

\section{REFERÊNCIAS}

Alves, R. J. V., L. Cardin \& M . S. Kropf. 2007. Angiosperm disjunction "campos rupestres restingas": a re-evaluation. Acta Bot. Brasil. 21: 675-685.

APG (Angiosperm Phylogeny Group). 2016. $\mathrm{Na}$ update of the Angiosperm Phylogeny Group classification for the orders and families of flowering plants: APG IV. Bot. J. Linn. Soc. 181: 1-20.

Araujo, D. S. D. 2000. Análise florística e fitogeográfica das Restingas do estado do Rio de Janeiro. Tese (Doutorado em Ecologia), Rio de Janeiro, Universidade Federal do Rio de Janeiro.

Araujo, D. S. D, M. C. A. Pereira \& M. C. P. Pimentel. 2004. Flora e estrutura de comunidades na Restinga de Jurubatiba síntese dos conhecimentos com enfoque especial para a formação aberta de Clusia, p.
59-76. In: C.F.D. Rocha, F.A. Esteves \& F.R. Scarano (orgs.). Pesquisas de longa duração na Restinga de Jurubatiba: ecologia, história natural e conservação. São Carlos: RiMa.

Assis, A. M., L. D. Thomaz \& O. J. Pereira. 2004. Florística de um trecho de floresta de restinga no município de Guarapari, Espírito Santo, Brasil. Acta Bot. Brasil. 18: 191-201.

Bigarella, J. J. \& D. Andrade-Lima. 1982. Paleoenvironmental changes in Brazil, p. 2740. In: G.T. Prance (ed.). Biological diversification in the Tropics. Plenum Press, New York.

BFG - The Brazil Flora Group. 2015. Growing knowledge: an overview of Seed Plant diversity in Brazil. Rodriguésia. 66: 1085-1113.

Cantarelli, J. R. R., E. B. Almeida-Jr, F. S. Santos-Filho \& C. S. Zickel. 2012. Tipos fitofisionômicos e florística da restinga da APA de Guadalupe, Pernambuco, Brasil. INSULA Rev. Bot. 41: 95-117.

Carvalho, D. A. \& C. F. C. Sá. 2011. Estrutura do estrato herbáceo de uma restinga arbustiva aberta na APA de Massambaba, Rio de Janeiro, Brasil. Rodriguésia 62: 367-378.

Castelo, A. J. \& J. M. A. Braga. 2017. Checklist of sand dune vegetation on the tropical southeastern Brazil coast. Check List. 13: 2058.

Colodete, M. F. \& O. J. Pereira. 2007. Levantamento florístico da restinga de Regência, Linhares/ES. R. bras. Bioci. 5: 558-560.

Costa, G. M., W. M. B. São-Mateus, R. P. Oliveira \& A. M. Giulietti. 2010. Flora da Bahia: Bonnetiaceae. SCB 10:77-79.

Fabris, L. C. \& O. J. Pereira. 1998. Florística da formação pós-praia, na restinga de Setiba, município de Guarapari (ES). In: Simpósio de Ecossistemas Brasileiros, 4., Águas de Lindóia. Anais... São Paulo: ACIESP. v.3, p.165-176.

Fanzeres, A. 2003. RESTINGAS: CONHECER PARA PRESERVAR. Ciência Hoje on-line. Disponível em: < http://cienciahoje.org.br/ restingas-conhecer-para-preservar/> . Acesso em 26 de junho de 2018.

Fernandes, M. F. \& L. P. Queiroz. 2015. Floristic surveys of Restinga Forests in southern Bahia, Brazil, reveal the effects of geography on community composition. Rodriguésia. 66: 051-073. 
Fiaschi, P. \& J. R. Pirani. 2009. Review of plant biogeographic studies in Brazil. J. Syst. Evol. 47: 477-496.

Flora do Brasil 2020 em construção. Jardim Botânico do Rio de Janeiro. Disponível em: < http://floradobrasil.jbrj.gov.br/ >. Acesso em 23 de Agosto de 2018.

Harley, R. M. 1995. Introdução, p. 1-40. In: B.L. Stannard (Ed.). Flora of the Pico das Almas, Chapada Diamantina, Bahia, Brazil. Kew, Royal Botanic Gardens.

Hammer, Ø., D. A. T. Harper, P. D. Ryan. 2001. PAST: Paleontological statistics software package for education and data analysis. Palaeontol. Electronica. 4: 9.

Hammerton, J. 2001. Casuarinas in The Bahamas: a clear and present danger. Bahamas J. of Science. 9:2-14.

Kendall, P \& B. Snelson. 2009. The role of floristic survey data and quantitative analysis in identification and description of ecological communities under threatened species legislation: A case study from north-eastern New South Wales. Ecol. Managem. Restorat. 10: 16-26.

Lourenco, A. R. L. \& M. R. V. Barbosa. 2012. Myrtaceae in the restinga vegetation at the northern limit of the Atlantic coastal forest, Brazil. Rodriguésia. 63: 373-393.

Magnago, L. F. S., S. V. Martins, C. E. G. R., Schaefer \& A. V. Neri. 2010. Gradiente fitofisionômico-edáfico em formações florestais de Restinga no Sudeste do Brasil. Acta Bot. Brasil. 24: 734-746.

Magnago, L. F. S., O. J. Pereira, \& S. V. Martins. 2011a. Caracterização das formações ribeirinhas na restinga do Parque Natural Municipal de Jacarenema, Vila Velha, ES, Brasil, p. 415-440. In: Felfili J.M., P.V. Eisenlohr, M.M.R.F. Melo, L.A. Andrade \& J.A.A. Meira-Neto. (Org.). Fitossociologia no Brasil: métodos e estudos de caso. Viçosa, Editora da Universidade Federal de Viçosa.

Magnago, L. F. S., S. V. Martins \& O. J. Pereira. 2011b. Heterogeneidade florística das fitocenoses de restingas nos estados do Rio de Janeiro e Espírito Santo, Brasil. Revista Árvore 35: 245-254.

Magurran, A. E. 2013. Medindo a Diversidade Biológica. Editora da UFPR, Curitiba.

Martinelli, G. 2010. Conhecer a Flora para Protegê-la. Sci. Amer. 39: 36-41.
Martins, S. E., L. Rossi, P. S. P. Sampaio \& M. A. G. Magenta. 2008. Caracterização florística de comunidades vegetais de restinga em Bertioga, SP. Brasil. Acta Bot. Brasil. 22: 249-274.

Martins, M. L. L. 2012. Fitofisionomia das formações vegetais da Restinga da Área de Proteção Ambiental (APA) de Guaibim, Valença, Bahia, Brasil. R. bras. Bioci. 10: 66-73.

Menezes, C. M., F. D. Santana, V. S. Silva, V. I. S. \& D. S. Araújo. 2012. Florística e fitossociologia em um trecho de restinga no Litoral Norte do Estado da Bahia. Biotemas .25: 31-38.

Meira-Neto, J. A. A., A. L. Souza, J. M. Lana \& Valente, G. E. 2005. Composição florística, espectro biológico e fitofisionomia da vegetação de Muçununga nos municípios de Caravelas e Mucuri, Bahia.

Revista Árvore. 29: 139-150.

Monteiro, M.M., A. Giaretta, O.J. Pereira \& L. F. T. Menezes. 2014. Composição e estrutura de uma restinga arbustiva aberta no norte do Espírito Santo e relações florísticas com formações similares no sudeste do Brasil. Rodriguésia 65: 61-72.

Moraes, J. N. \& M. A. G. Magenta. 2014. Levantamento Florístico de um fragmento de Restinga no município de Santos adjacente ao Rio Diana no estado de São Paulo. Unisanta BioScience. 3: 115-121.

Mori, S. A., L. A. M. Silva, G. Lisboa \& L. Coradin. 1989. Manual de manejo do herbário fanerogâmico. Centro de Pesquisa do Cacau, Ilhéus. Pp. 1-104

Moro, M. F \& F. R. Martins. 2011. Métodos de levantamento do componente arbóreo-arbustivo, p. 174-212. In: J.M. Felfili, P.V. Eisenlohr, M.M.R.F. Melo, L.A. Andrade \& J.A.A. Meira-Neto. (Org.). Fitossociologia no Brasil: métodos e estudos de caso. Viçosa, Editora da Universidade Federal de Viçosa.

Myers, N., R. A. Mittermeier, C. G. Mittermeier, G. A. da Fonseca \& J. Kent. 2000. Biodiversity hotspots for conservation priorities. Nature, 403: 853-858. 
Pereira, M. C. A., D. S. D. Araújo \& O. J. Pereira. 2001. Estrutura de uma comunidade arbustiva da restinga de Barra de Maricá RJ. Revista Brasil. Bot. 24: 273-281.

Pereira, O. J., L. D. Thomaz \& D. S. D. Araujo. 1992. Fitossociologia da vegetação de ante-dunas da restinga de Setiba/Guarapari e em Interlagos/Vila Velha; ES. Bol. Mus. Biol. Mello Leitão. 1: 65-75.

Pereira, O. J. \& D. S. D. Araujo. 1995. Estrutura da vegetação de entre moitas da formação aberta de Ericaceae no Parque Estadual de Setiba, ES, p. 245-257. In: Esteves F. A. (Org.). Oecologia Brasiliensis: estrutura, funcionamento e manejo de ecossistemas brasileiros. Rio de Janeiro, UFRJ.

Queiroz E. P., D. B. O. S. Cardoso \& M. H. S. Ferreira. 2012. Composição floristica da vegetação de resting da APA Rio Capivara, Litoral Norte da Bahia, Brasil. SCB. 12: 119141.

Rocha, C. F. D., H. G. Bergallo, M. Van Sluys, M. A. S. Alves \& C. E. Jamel. 2007. The remnants of restinga habitats in the brazilian Atlantic Forest of Rio de Janeiro state, Brazil: habitat loss and risk of disappearance. Brazil. J. Biol. 67: 263-273.

Rodgers III, J. C. \& S. Ambinakudige. 2012. Distribution Patterns of Invasive Casuarinas (Casuarina equisetifolia L.) Within Beach Environments on San Salvador Island, the Bahamas. Nat. Area J. 32: 386-390.

Roque, N. \& V. C. Carvalho. 2011. Estudos taxonômicos do gênero Calea (Asteraceae, Neurolaeneae) no estado da Bahia, Brasil. Rodriguésia. 62: 547-561.

Santos, A. P. B., F. S. Espírito-Santo \& A. Rapini. 2015. Flora da Bahia: Calophyllaceae. SCB 15: 10.13102/scb884

Santos, A. P. B., C. Bitencourt \& A. Rapini. 2016. Distribution patterns of Kielmeyera (Calophyllaceae): the Rio Doce basin emerges as a confluent area between the northern and southern Atlantic Forest. Neotropical Biodiversity. 3:1-9.

Santos-Filho, F. S. D. 2009. Composição florística e estrutural da vegetação de restinga do Estado do Piauí. Tese (Doutorado em Botânica), Recife, Universidade Federal Rural de Pernambuco.

Scarano, F. R. 2002. Structure, function and floristic relationships of plants communities in stressful habitats marginal to the Brazilian Atlantic Rainforest. Ann. Bot. 90: 517-524.

Saporetti-Junior, A. W., C. E. G. R. Schaefer, A. L. de Souza, M. P. Soares, D. S. D. Araujo \& J. A. A. Meira-Neto. 2012. Influence of soil physical properties on plants of the Mussununga ecosystem, Brazil. Folia Geobot. 47: 29-39.

Specieslink. 2018. Disponível em: <http:// splink.cria.org.br/> Acesso em: 01 de junho de 2018.

Souza, M. C., M. P. Morim, M. M.S . Conde \& L. F. T. Menezes. 2007. Subtribo Myrciinae O. Berg (Myrtaceae) na Restinga da Marambaia, RJ, Brasil. Acta Bot. Brasil. 21: 49-63.

SEI (Superintendência de Estudos Econômicos e Sociais do Estado da Bahia). 2015. Banco de dados geo-ambientais. Disponível em <http://www.sei.ba.gov.br>. Acesso em 3 março 2015.

Swearingen, J. 2008. Casuarinas; Casuarina equisetifolia L. Plant Conservation Alliance's Alien Plant Working Group, U.S. National Parks Service. 